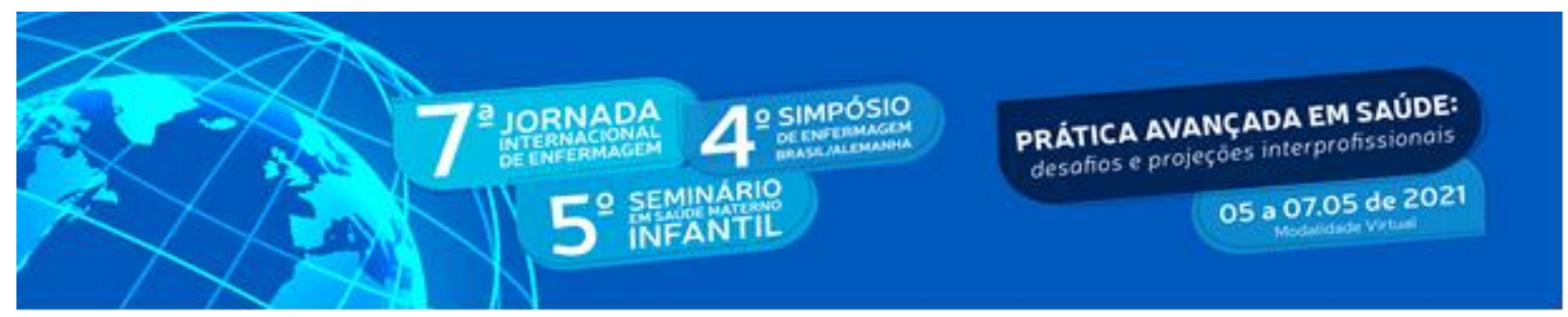

DOI: http://doi.org/10.48195/jie2021-122

\title{
SUPLEMENTAÇÃO PROFILÁTICA DE FERRO EM CRIANÇAS: UMA PESQUISA DOCUMENTAL
}

\section{Francisca Georgina Macedo de Sousa ${ }^{1}$; Gabrielle Pontes Santos ${ }^{2}$; Jéssica Nathália de Melo Sousa ${ }^{3}$; Nathália da Silva Licar ${ }^{4}$; Nayana de Paiva Fontenelle Xerez ${ }^{5}$; Vanessa Dias da Silva ${ }^{6}$}

\section{RESUMO}

Objetivo: Caracterizar a situação da suplementação profilática de ferro em crianças a partir dos 4 meses de idade. Metodologia: Estudo documental quantitativo tendo como contexto os registros dos profissionais na Caderneta de Saúde da Criança. Resultado: Das 1603 CSC analisadas, 112 (6,98\%) havia registro da suplementação de ferro e em 1491 (93,01\%) delas não havia registro dessa informação. Constatou-se que em 3 Cadernetas $(0,18 \%)$ a suplementação de ferro foi iniciada aos 4 meses de idade, $32(1,99 \%)$ com 6 meses e $77(4,80 \%)$ após 6 meses de vida. Conclusão: A anemia ferropriva pode desencadear problemáticas no desenvolvimento da criança, principalmente em idade pré-escolar, fazendo-as vulneráveis a possíveis infecções devido à baixa no seu sistema imunológico. Sendo assim, ratifica-se a importância do enfermeiro assegurar a suplementação de ferro a todas as crianças quando das consultas ambulatoriais nos serviços de atenção básica até os dois anos de idade.

Palavras-chave: Anemia ferropriva; Sulfato ferroso; Micronutrientes; Saúde da criança.

\footnotetext{
ABSTRACT

Objective: To characterize the situation of prophylactic iron supplementation in children from 4 months of age. Methodology: Quantitative documentary study with the records of professionals in the Child Health Handbook as a context. Result: Of the 1603 CSCs analyzed, 112 (6.98\%) had a record of iron supplementation and in $1491(93.01 \%)$ of them there was no record of this information. It was found that in 3 passbooks $(0.18 \%)$ iron supplementation was started at 4 months of age, $32(1.99 \%)$ at 6 months and $77(4.80 \%)$ after 6 months of life. Conclusion: Iron deficiency anemia can trigger problems in the child's development, especially at preschool age, making them vulnerable to possible

${ }^{1}$ Enfermeira. Docente do Departamento de Enfermagem da UFMA. Líder do Grupo de Ensino e Pesquisa na Saúde da Família, Criança e Adolescente - GEPSFCA. Coordenadora da Pesquisa. E-mail: francisca.gms@ufma.br

2 Discente do Curso de Graduação em Enfermagem da UFMA. Membro do GEPSFCA. E-mail: gabriellepontessp@gmail.com

3 Discente do Curso de Residência Multiprofissional HUUFMA na Área Saúde da Criança. Membro do GEPSFCA. E-mail: jessica-nathy@ hotmail.com

${ }^{4}$ Discente do Curso de Graduação em Enfermagem/CEST. Membro do GEPSFCA. E-mail: nathalya.licar.nl@gmail.com

${ }^{5}$ Enfermeira. Nutricionista. Docente CEST. Orientadora. Membro do GEPSFCA. E-mail: nayana.fontenelle.nf@gmail.com

${ }^{6}$ Discente do Curso de Graduação em Enfermagem da UFMA. Membro do GEPSFCA. E-mail: vanessad25978@gmail.com
} 


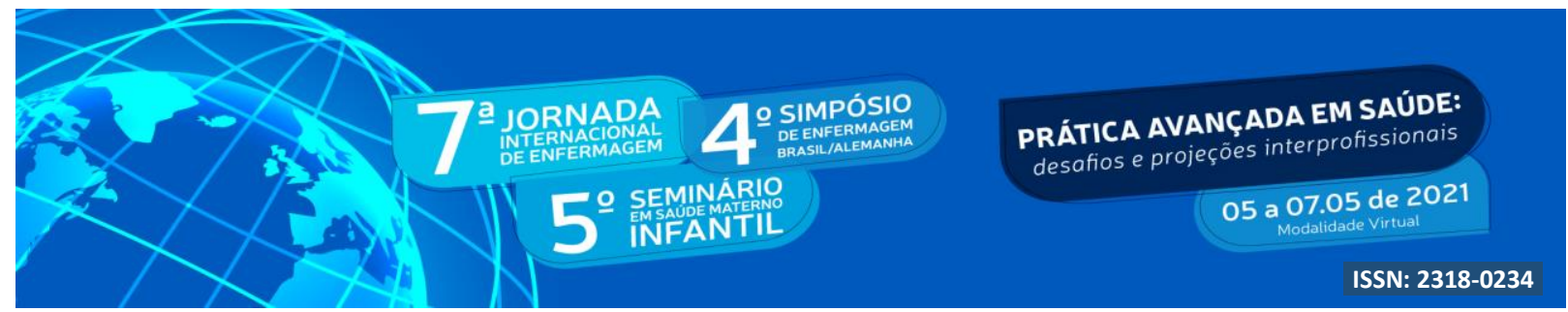

infections due to low immune system. Thus, the importance of nurses to ensure iron supplementation is confirmed for all children during outpatient consultations in primary care services up to the age of two.

Keywords: Iron deficiency anemia; Ferrous sulphate; Micronutrients; Child health.

\section{INTRODUÇÃO}

A anemia por deficiência de ferro (ADF) apresenta-se como situação agravante de caráter mundial. De acordo com o Ministério da Saúde (MS) estima-se que cerca de 90\% da anemia é causada pela carência de ferro, tendo como maior susceptibilidade crianças menores de dois anos de idade, gestantes e lactantes. Uma vez que há carência de micronutrientes essenciais, como o sulfato ferroso, tornam-se maiores as chances para possíveis manifestações de infecção devido à baixa do sistema imunológico, podendo assim, afetar diretamente na execução do desenvolvimento cognitivo, comportamental e crescimento da criança (GONTIJO et al., 2017).

Posto que há uma grande predominância da anemia no Brasil, foram levantados dados em conformidade com a Pesquisa Nacional de Demografia e Saúde da Criança e da Mulher (PNDS), demonstrando maior incidência em crianças menores de cinco anos com 20,9\% em todo país, sabendo que $24,1 \%$ estende-se a crianças menores de dois anos de idade. Havendo assim, um aumento substancial na população em idade pré-escolar (BRASIL, 2013).

Evidencia-se que o público infantil demonstra maior prevalência na vulnerabilidade quanto a anemia ferropriva, sendo não somente uma preocupação da Saúde Pública, bem como, pode estar relacionado ao acentuado crescimento junto ao seu estado nutricional. Dessa forma, é crucial que a criança receba uma demanda satisfatória de micronutrientes através da ingestão de alimentos à base de ferro, possibilitando um desenvolvimento qualitativo que seja suficiente, a fim, de evitar o surgimento dessa e outras patologias (ANDRÉ et al., 2018).

Constata-se que para garantia de uma melhor qualidade de vida e o bem estar biopsicossocial da criança, estão interligados fatores extrínsecos, como o âmbito social, estado nutricional e medidas de cuidados adequados. Aponta-se que os indícios de má nutrição e a falta de absorção de micronutrientes acarretará posteriormente na condição física da criança. Isso ocorre, por intermédio de uma dieta inapropriada e com pouca diversificação de alimentos (VAZ et al., 2017). 


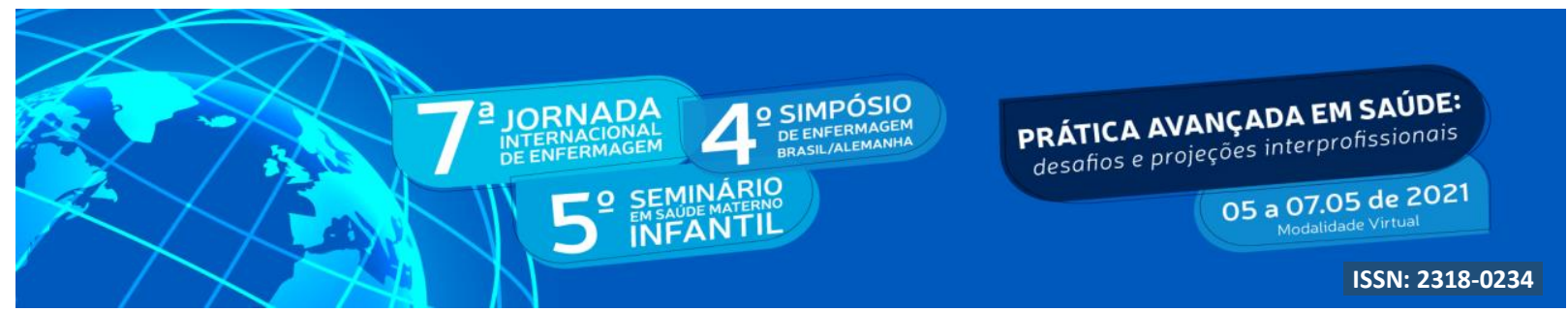

Diante das medidas adotadas pelo Ministério da Saúde (MS) para atenuar os impactos provocados pela deficiência ferropriva, foram instituídas estratégias de suplementação profilática de ferro, dentre essas intervenções dar-se menção ao Programa Nacional de Suplementação de Ferro (PNSF), criado com o intuito de melhorar exponencialmente a situação de ferro nos primeiros anos de vida. Por conseguinte, cria-se a fortificação dos alimentos preparados para as crianças com inserção de micronutrientes em pó (NUTRISUS), tal como, a promoção da alimentação adequada e saudável, empenhando-se em agregar cada vez mais alimentos ricos em ferro na dieta infantil.

Como identificação documental da criança dispõe-se da Caderneta de Saúde da Criança (CSC), que caracteriza-se como principal instrumento de promoção integrada à saúde, pois nela integram-se informações pertencentes ao usuário. Por sua vez, aprimorando o atendimento por parte dos profissionais que fazem o acompanhamento da criança, e é de responsabilidade dos mesmos o preenchimento correto das ações definidas na Política Nacional de Atenção Integral à Saúde da Criança (PNAISC), assegurando atualização rotineiramente de seu conteúdo, tendo como exemplo, os indicadores de crescimento qualificados pelo perímetro cefálico, peso, altura e Índice de Massa Corporal (IMC), nas curvas de crescimento, avaliação dos marcos do desenvolvimento, o calendário básico de vacinação, a suplementação de ferro e de vitamina A, além de outras ações (BRASIL, 2018).

\section{OBJETIVO}

Caracterizar a situação da suplementação profilática de ferro em crianças a partir dos 4 meses de idade conforme os registros na Caderneta de Saúde da Criança em São Luís - MA.

\section{METODOLOGIA}

Trata-se de pesquisa documental com abordagem quantitativa onde foram avaliados os registros realizados pelos profissionais da Atenção Primária em Saúde de São Luís - MA na Caderneta de Saúde da Criança (CSC), no que tange a situação de suplementação profilática de ferro. 


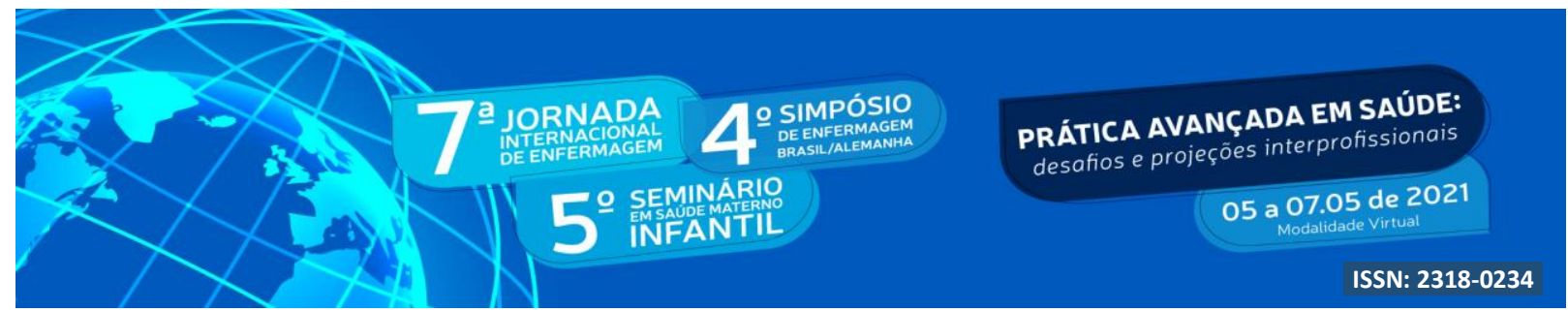

Salienta-se que esta pesquisa vincula-se a um projeto maior "Caderneta de Saúde da Criança e o Manejo Profissional das Ações de Saúde”, que visa identificar como as ações de saúde da criança são registradas na CSC, vinculado ao Departamento de Enfermagem da Universidade Federal do Maranhão - UFMA e ao Grupo de Estudo e Pesquisa na Saúde da Família, Criança e Adolescente - GEPSFCA e foi submetida a Plataforma Brasil com o CAAE n 17772819.1.0000.5087, no qual recebeu parecer de aprovação.

Para acesso às CSC, utilizou-se o espaço das salas de imunização das Unidades Básicas de Saúde (UBS) do município de São Luís, capital do Estado do Maranhão. Para seleção das UBS's considerou-se aquelas que haviam realizado 1000 ou mais atendimentos pediátricos ambulatoriais no ano de 2019. Sendo assim, das 57 UBS's do município, 31 delas participaram da pesquisa.

A amostra do estudo é de 2.305 crianças, no entanto, os resultados aqui apresentados são parciais, tendo em vista a não conclusão da coleta dos dados em função do período pandêmico que assola todo o país. Portanto, dadas as circunstâncias, utilizou-se a amostra parcial de 1889 CSC, destas 286 são de crianças menores de 4 meses que foram excluídas, pois o início da suplementação de ferro se dá a partir do 4 ou sexto mês conforme recomendações do Ministério da Saúde (MS). Sendo assim, para a pesquisa considerou-se todas as crianças a partir dos 4 meses de idade que receberam a suplementação, totalizando 1603 CSC analisadas. A coleta de dados se restringiu às páginas de identificação da criança e a de registro de suplementação de ferro nas CSC de crianças atendidas nas Unidades Básicas de Saúde do município de São Luís - Maranhão.

A avaliação dos dados da pesquisa foi apoiada nas recomendações do Ministério da Saúde (MS), conforme mostra o quadro abaixo:

Quadro 1 - Recomendações quanto à suplementação de ferro

\begin{tabular}{|c|c|}
\hline Situação & Recomendação \\
\hline $\begin{array}{l}\text { Recém-nascidos a termo, de peso adequado } \\
\text { para idade gestacional em aleitamento } \\
\text { materno exclusivo ou não }\end{array}$ & $\begin{array}{l}1 \mathrm{mg} \text { de ferro elementar } / \mathrm{kg} \text { peso/dia a partir } \\
\text { do quarto mês (ou da introdução de outros } \\
\text { alimentos) até o } 24^{\circ} \text { mês de vida. }\end{array}$ \\
\hline $\begin{array}{l}\text { Recém-nascidos a termo, de peso adequado } \\
\text { para idade gestacional em uso de menos de } \\
500 \mathrm{~mL} \text { de fórmula infantil por dia }\end{array}$ & $\begin{array}{l}1 \mathrm{mg} \text { de ferro elementar } / \mathrm{kg} \text { peso/dia a partir } \\
\text { do quarto mês (ou da introdução de outros } \\
\text { alimentos) até o } 24^{\circ} \text { mês de vida. }\end{array}$ \\
\hline
\end{tabular}




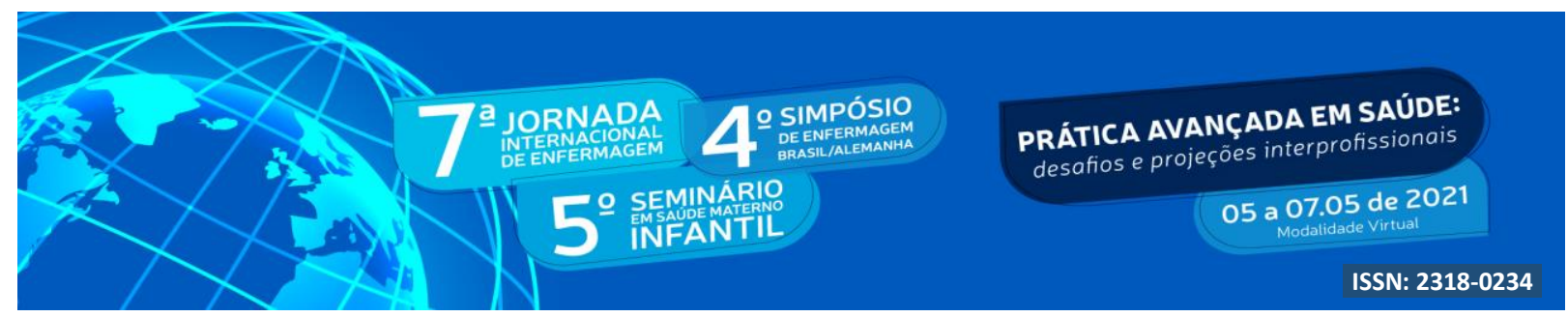

\begin{tabular}{|c|c|}
\hline $\begin{array}{l}\text { Recém-nascidos a termo com peso inferior a } \\
2.500 \mathrm{~g}\end{array}$ & $\begin{array}{l}2 \mathrm{mg} / \mathrm{kg} \text { peso/dia durante um ano. Após este } \\
\text { período, } 1 \mathrm{mg} / \mathrm{kg} \text { peso/dia por mais um ano. }\end{array}$ \\
\hline $\begin{array}{l}\text { Recém-nascidos pré-termo e recém-nascido } \\
\text { com peso entre } 2.500 \mathrm{~g} \mathrm{a} 1.500 \mathrm{~g}\end{array}$ & $\begin{array}{l}2 \mathrm{mg} / \mathrm{kg} \text { peso/dia durante um ano. Após este } \\
\mathrm{prazo}, 1 \mathrm{mg} / \mathrm{kg} \text { peso/dia por mais um ano. }\end{array}$ \\
\hline $\begin{array}{l}\text { Recém-nascidos pré-termo com peso entre } \\
1.500 \text { e } 1.000 \mathrm{~g}\end{array}$ & $\begin{array}{l}3 \mathrm{mg} / \mathrm{kg} \text { peso/dia durante um ano. Após este } \\
\text { período, } 1 \mathrm{mg} / \mathrm{kg} \text { peso/dia por mais um ano. }\end{array}$ \\
\hline $\begin{array}{l}\text { Recém-nascidos pré-termo com peso } \\
\text { inferior a } 1.000 \mathrm{~g}\end{array}$ & $\begin{array}{l}4 \mathrm{mg} / \mathrm{kg} \text { peso/dia durante um ano. Após este } \\
\text { período, } 1 \mathrm{mg} / \mathrm{kg} / \text { dia por mais um ano. }\end{array}$ \\
\hline
\end{tabular}

Fonte: Ministério da Saúde, 2013.

Diante das informações contidas no esquema acima, observa-se que a suplementação de ferro deve estar pautada em alguns critérios pertinentes ao que deve ser recomendado à criança, considerando-se a idade gestacional, o peso do nascimento e a condição do aleitamento materno, se exclusivo ou alimentação complementar. Partindo desse pressuposto, o início da suplementação de ferro ocorre no quarto ou no sexto mês a depender dos critérios acima referidos e se prolonga até o $24^{\circ}$ mês de vida.

Dito isto, o Ministério da Saúde (MS) enfatiza que a reserva de ferro contida no aleitamento materno exclusivo, nos seis primeiros meses de idade, é suficiente para manutenção das necessidades fisiológicas, não precisando fazer uso de qualquer outro completo nutricional. Não obstante a isso, entre os quatro e seis primeiros meses de vida ocorre gradativamente o esgotamento da reserva de ferro, fazendo com que a criança necessite da complementação por meio de alimentos tendo como destaque na incumbência de nutrientes. Com isso, configura-se que o consumo do ferro seja adequado para a demanda implícita para essa fase etária.

De posse destas assertivas, questiona-se: Qual a situação da suplementação de ferro profilático de crianças atendidas nos serviços de puericultura das Unidades Básicas de Saúde?

Os resultados da pesquisa permitirão reflexão crítica sobre o acesso das crianças às ações definidas pela PNAISC assim como o manejo dessas informações e os respectivos registros dos profissionais na CSC. A relevância do estudo se estende, portanto, à qualificação da atenção à criança a partir das determinações da PNAISC assim como o manejo da CSC. 


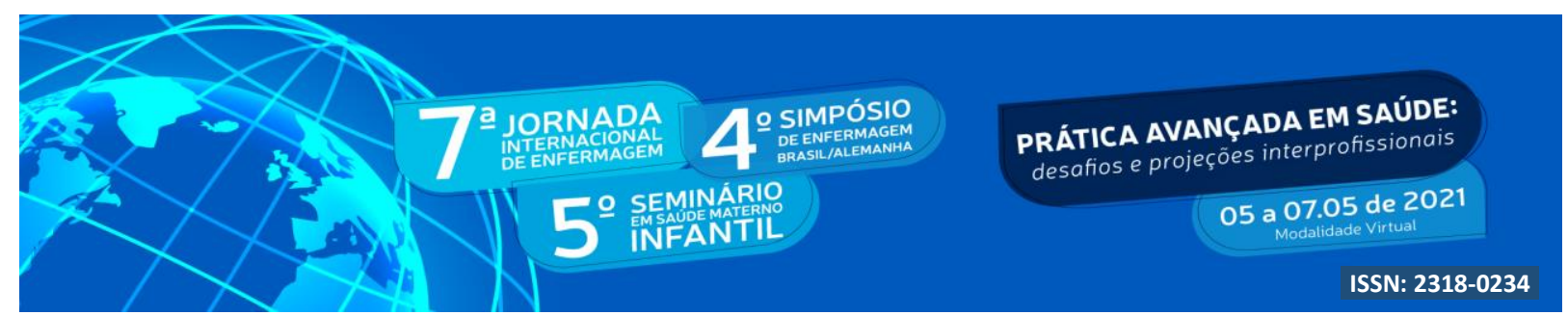

\section{RESULTADOS E DISCUSSÃO}

Tabela 1 - Registro de suplementação de ferro nas Cadernetas de Saúde da Criança. São Luís - MA $2019 / 2020$

\begin{tabular}{|l|c|c|}
\hline \multicolumn{1}{|c|}{ Caracterização } & N & \% \\
\hline Com registro de suplementação de ferro profilático & 112 & $6,98 \%$ \\
\hline Sem registro de suplementação & 1491 & $93,01 \%$ \\
\hline Total & $\mathbf{1 6 0 3}$ & $\mathbf{1 0 0 \%}$ \\
\hline
\end{tabular}

Fonte: Dados da pesquisa.

Verificou-se que das 1603 CSC, 112 (6,98\%) apresentaram registros do esquema de suplementação de ferro profilático. Entretanto, 93,01\% das CSC não haviam nenhum registro quanto a esta ação de prevenção de anemia por deficiência de ferro. Esta condição pode revelar duas situações: a primeira, é que um percentual muito baixo de crianças não tiveram acesso a suplementação de ferro em tempo oportuno; a segunda, como o estudo foi documental, não se pode afirmar que as crianças sem registro da referida suplementação não receberam a dose de ferro. Mas, a pesquisa revelou, enfaticamente, que o manejo das ações da criança definidas pela PNAISC assim como o respectivo registro na CSC são inadequados e insuficientes.

Tabela 2 - Início da suplementação por faixa etária de acordo com registros na CSC. São Luís - MA,

\begin{tabular}{|l|c|c|}
\hline \multicolumn{1}{|c|}{ Faixa etária } & 2019/2020 & \% \\
\hline Iniciada com 4 meses de idade & 03 & $0,18 \%$ \\
\hline Iniciada com 6 meses de idade & 32 & $1,99 \%$ \\
\hline Iniciada após 6 meses de idade & 77 & $4,80 \%$ \\
\hline Não há registro & 1491 & $93,01 \%$ \\
\hline Total & $\mathbf{1 6 0 3}$ & $\mathbf{1 0 0 \%}$ \\
\hline
\end{tabular}

Fonte: Dados da pesquisa.

Das CSC com registros de suplementação, três $(0,18 \%)$ delas foram iniciadas aos 4 meses de idade e $32(1,99 \%)$ aos 6 meses. Portanto, o maior percentual de crianças iniciaram 


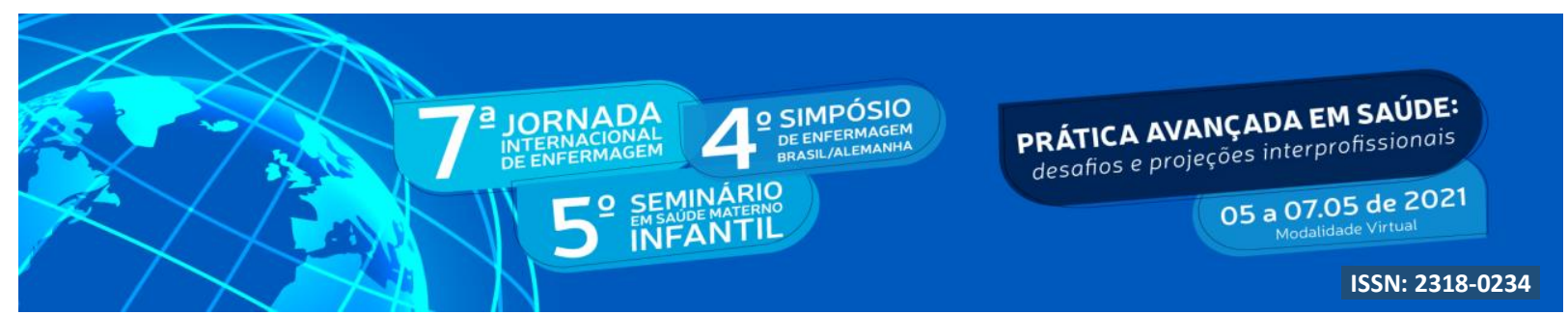

a suplementação após a idade recomendada (4,80\%) o que concorre para maior vulnerabilidade da saúde infantil.

\section{CONCLUSÃO}

O estudo revelou número insignificante de registro de suplementação de ferro na CSC. Entretanto, não se pode afirmar que as crianças não fizeram a suplementação, mas que o manejo no registro das ações da criança definidas na PNAISC não recebe a devida atenção pelos profissionais da Atenção Primária em Saúde.

O adequado registro dessas ações na CSC deve ser valorizado por todos os profissionais independente do contexto de atuação, pois é através destes que é possível monitorar de maneira efetiva a saúde da criança. Por outro lado, assegurar o acesso da criança a todas as ações de promoção da saúde e de prevenção de agravos configura-se como atitude e compromisso ético e técnico daqueles envolvidos com a atenção à criança.

A deficiência de ferro, assim como a anemia por deficiência de ferro são agravos prevalentes em algumas regiões brasileiras, dentre as quais a região Nordeste. É nesse contexto situacional, que a suplementação de ferro deve ser assegurada, cabendo ao profissional a orientação e a instituição da suplementação de ferro minimizando as falhas existentes para o controle da deficiência desse micronutriente.

\section{REFERÊNCIAS}

ANDRÉ, Hercilio Paulino et al. Indicadores de insegurança alimentar e nutricional associados à anemia ferropriva em crianças brasileiras: uma revisão sistemática. Ciência \& Saúde Coletiva, v. 23, p. 1159-1167, 2018. Disponível em: https://www.scielo.br/pdf/csc/v23n4/1413-8123-csc-23-04-1159.pdf. Acesso em: 07 mar. 2021.

BRASIL. Ministério da Saúde. Política Nacional de Atenção Integral à Saúde da Criança: orientações para implementação. 2018. Disponível em: https://portaldeboaspraticas.iff.fiocruz.br/wp-content/uploads/2018/07/Pol\%C3\%ADticaNacional-de-Aten\%C3\%A7\%C3\%A3o-Integral-\%C3\%A0-Sa\%C3\%BAde-daCrian\%C3\%A7a-PNAISC-Vers\%C3\%A3o-Eletr\%C3\%B4nica.pdf. Acesso em: 07 mar. 2021.

BRASIL. Ministério da Saúde. Secretaria de Atenção à Saúde. Departamento de Atenção Básica. Programa Nacional de Suplementação de Ferro: manual de condutas gerais / Ministério da Saúde. Secretaria de Atenção à Saúde. Departamento de Atenção Básica. 


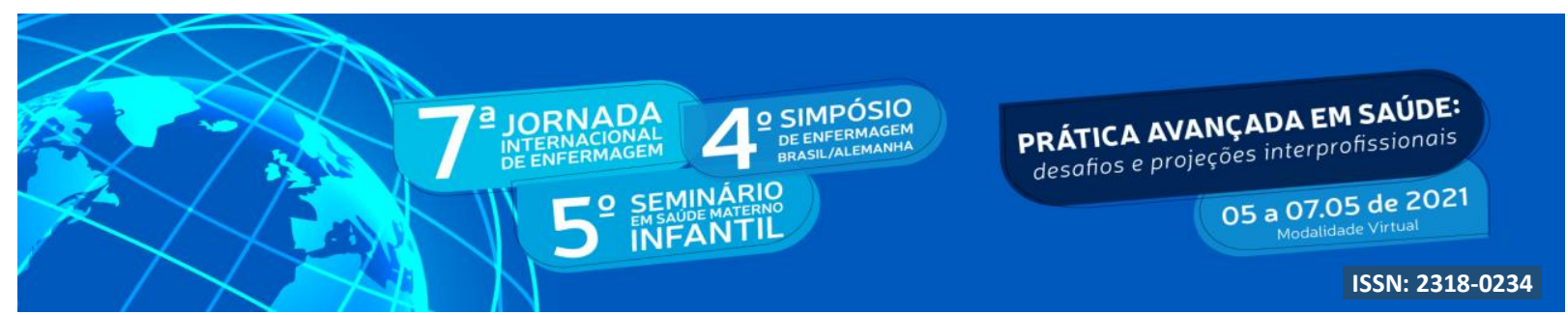

Brasília: Ministério da Saúde, 2013. Disponível em:

http://bvsms.saude.gov.br/bvs/publicacoes/manual_suplementacao_ferro_condutas_gerais.pdf . Acesso em: 07 mar. 2021.

GONTIJO, Tarcísio Laerte et al. Prática profilática da anemia ferropriva em crianças na estratégia saúde da família. Revista de Enfermagem do Centro-Oeste Mineiro, v. 7, 2017. Disponível em: http://seer.ufsj.edu.br/index.php/recom/article/view/1204/1555. Acesso em: 07 mar. 2021.

VAZ, Monique Almeida et al. Suplementação na infância e a prevenção da carência de micronutrientes: Artigo de revisão. Revista de Medicina e Saúde de Brasília, v. 6, n. 1, 2017. Disponível em: https://portalrevistas.ucb.br/index.php/rmsbr/article/view/7684/5108. Acesso em: 07 mar. 2021. 